\title{
Evaluation of the Reverse Transcription Loop-Mediated Isothermal Amplification (RT-LAMP) as a Screening Method for the Detection of Influenza Viruses in the Fecal Materials of Water Birds
}

\author{
Hiromi YOSHIDA ${ }^{1)}$, Yoshihiro SAKODA ${ }^{1)}$, Mayumi ENDO ${ }^{1)}$, Masayuki MOTOSHIMA ${ }^{1)}$, Fumi YOSHINO ${ }^{1)}$, \\ Naoki YAMAMOTO ${ }^{1)}$, Masatoshi OKAMATSU ${ }^{1)}$, Takahiro SOEJIMA ${ }^{2)}$, Syouhei SENBA ${ }^{2)}$, Hidetoshi KANDA ${ }^{2)}$ and \\ Hiroshi $\mathrm{KIDA}^{2-4) *}$ \\ ${ }^{1)}$ Laboratory of Microbiology, Department of Disease Control, Graduate School of Veterinary Medicine, Hokkaido University, Kita 18 \\ Nishi 9, Sapporo 060-0818, ${ }^{2}$ Eiken Chemical Company, Limited, 1381-3 Shimoishigami, Ohtawara, Tochigi 324-0036, ${ }^{3)}$ Research \\ Center for Zoonosis Control, Hokkaido University, Kita 20 Nishi 10, Sapporo 001-0020 and ${ }^{4)}$ Japan Science and Technology Agency \\ Basic Research Programs, 5 Sanbancho, Chiyoda-ku, Tokyo 102-0075, Japan
}

(Received 12 November 2010/Accepted 14 January 2011/Published online in J-STAGE 28 January 2011)

\begin{abstract}
Migratory water birds are a natural reservoir for influenza A viruses. Viruses replicate in the intestines of ducks and are shed with the fecal materials. Virus isolation from collected fecal materials, therefore, is an integral part of the surveillance of avian influenza in water birds. In the present study, reverse transcription loop-mediated isothermal amplification (RT-LAMP) was assessed for its usefulness in detecting the RNA of influenza A viruses in fecal materials. It was found that, RT-LAMP specifically and sensitively detects the matrix gene of influenza A viruses. Influenza A viruses were isolated from the fecal materials in which viral RNA were detected by RT-LAMP in $35 \mathrm{~min}$. The present findings indicate that RT-LAMP is useful as a high throughput screening method for field samples prior to virus isolation, allowing the processing of hundreds of samples per day.

KEY WORDS: fecal material, influenza virus, reverses transcription loop-mediated isothermal amplification (RT-LAMP), screening, surveillance.
\end{abstract}

Influenza A viruses originate from non-pathogenic viruses that are circulating in migratory water birds, especially in ducks, and their nesting lake water [18, 29]. Influenza A viruses have two surface glycoproteins, the hemagglutinin (HA) and the neuraminidase (NA), and are further divided into H1-16 and N1-9 subtypes based on their antigenic specificity [5]. Each of the known subtypes of influenza A viruses has been isolated from ducks $[16,17]$. In summer, ducks are orally infected with influenza viruses by waterborne transmission at their nesting lakes close to the Arctic Circle in Siberia, Alaska, or Canada. The viruses replicate in the columnar epithelial cells that form crypts in the colon and are shed with fecal materials of ducks which do not show disease signs [18]. However, highly pathogenic avian influenza viruses (HPAIVs) replicate in multiple tissues and lead to a high mortality in chickens. HPAIVs are selected during multiple transmissions in chicken populations $[1,10,13,15,25]$. When highly pathogenic avian influenza (HPAI) outbreaks occur in poultry, depopulation of affected flocks is the standard strategy for the control of HPAI. In some Asian countries, however, HPAI is not effectively controlled within poultry and often spread over the environment. Therefore, ducks are infected with

\footnotetext{
* Correspondence to: KidA, H., Laboratory of Microbiology, Department of Disease Control, Graduate School of Veterinary Medicine, Hokkaido University, Kita 18 Nishi 9, Sapporo 0600818, Japan. e-mail:kida@vetmed.hokudai.ac.jp
}

HPAIVs transmitted from poultry during overwintering in South East Asia and spread HPAIVs. Many of them are found dead on the way back to their northern territory in spring $[2,23,26]$.

The surveillance of avian influenza is important for the control of HPAI. Recently, the importance of the surveillance has been increasing, since H5N1 HPAIVs have spread in poultry and migratory birds in more than 60 countries [21]. We have been conducting surveillance of avian influenza in ducks for more than 30 years and have isolated more than 1,200 strains from the fecal materials of water birds in Japan, Alaska, Siberia, and Mongolia [14, 19, 22, 23].

Virus isolation using embryonated chicken eggs has been the most reliable and sensitive method for the diagnosis of influenza. However, it takes two or three days to isolate influenza virus using embryonated chicken eggs. Thus, nucleic acid amplification techniques such as a real-time PCR method [7], nucleic acid sequence-based amplification (NASBA) [4], self-sustained sequence replication (3SR) [6], and strand displacement amplification (SDA) [27, 28] have been developed. The loop-mediated isothermal amplification (LAMP) is one of the molecular methods to amplify the genome under isothermal conditions with high specificity, efficiency, and rapidity [20]. This method has been used for rapid detection of influenza virus genes [3, 11, 12, 24]. In the present study, we assessed sensitivity and specificity of the reverse transcription (RT)-LAMP of influenza virus detection from fecal materials of ducks. 


\section{MATERIALS AND METHODS}

Viruses: Sixteen influenza viruses of $\mathrm{H} 1$ to $\mathrm{H} 16$ subtypes and seven avian paramyxoviruses (APMVs) listed in Table 2, were used for the evaluation of specificity of RT-LAMP. A/duck/Hokkaido/38/2009 (H4N6), A/duck/Hokkaido/152/ 2009 (H4N6), and A/duck/Hokkaido/322/2009 (H4N6), which were isolated from fecal materials of ducks in this study, were used for the evaluation of specificity of RTLAMP. A/duck/Hokkaido/5/1977 (H3N2) [18] was also used to evaluate the sensitivity of RT-LAMP. All viruses were propagated in ten-day-old embryonated chicken eggs.

Isolation of influenza viruses from the fecal materials: A/ duck/Hokkaido/5/1977 (H3N2) was inoculated intranasally at $10^{7.0} 50 \%$ egg infective dose $\left(\right.$ EID $\left._{50}\right)$ into 4 -week-old ducks (Chelly Valley strain, Japan) and fecal materials were collected. We also collected 342 fecal materials from ducks during September to December in 2009 at Ono Pond of Hokkaido University, Sapporo, Japan. Each sample was added to transport medium containing minimum essential medium (Nissui, Tokyo, Japan), 10,000 U/m l penicillin G (Meiji Seika, Tokyo, Japan), $10 \mathrm{mg} / \mathrm{m} l$ streptomycin (Meiji Seika), $0.3 \mathrm{mg} / \mathrm{m} l$ gentamicin (Merck, Whitehouse station, NJ, U.S.A.), $250 \mathrm{U} / \mathrm{m} l$ nystatin (Sigma, St. Louis, MO, U.S.A.), and $0.5 \%$ bovine serum albumin fraction $\mathrm{V}$ (Roche, Basel, Switzerland) to yield a $10-20 \%$ suspension. The suspension was centrifuged at 2,000 rpm for $5 \mathrm{~min}$ at $4^{\circ} \mathrm{C}$. The supernatant was used for virus isolation and RT-LAMP.

RNA extraction and RT-LAMP: Viral RNA was extracted from $50 \mu l$ of supernatant of the fecal materials and the allantoic fluids using a MagMAX-96 AI/ND Viral RNA Isolation Kit (Applied Biosystems, Foster City, CA, U.S.A.), according to the manufacturer's protocol. These RNAs were eluted in a final volume of $50 \mu l$ of elution buffer. RT-LAMP was carried out using a Loopamp RNA Amplification Kit (Eiken Chemical, Co, Ltd., Tokyo, Japan) and the primer set of the matrix (M) gene for the evaluation of influenza A viruses provided by Eiken Chemical Co., Ltd. This primer set comprising two outer, two inner, and two loop primers, which recognizing eight distinct regions on the target sequence were chosen [20]. The locations, names, and sequences of the primers are given in Table 1. Five microliters of extracted RNA were added to $20 \mu l$ of reaction mixture. The amplification reaction was performed using a Loopamp Real-time turbidimeter (LA-320C; Eiken Chemical) at $62.5^{\circ} \mathrm{C}$ for $35 \mathrm{~min}$ and then at $80^{\circ} \mathrm{C}$ for $5 \mathrm{~min}$ to terminate the reaction. Amplification of viral RNA was determined by real-time turbidity detection using LA-320C software (Threshold Value: 0.05).

Evaluation of the diagnostic sensitivity of RT-LAMP: To evaluate the sensitivity of RT-LAMP, the detection limit of the assay was determined by serial two-fold dilutions of the allantoic fluid of the embryonated chicken egg infected with A/duck/Hokkaido/5/1977 (H3N2) and the supernatant of fecal materials collected from domestic ducks experimentally infected with A/duck/Hokkaido/5/1977 (H3N2). Viral RNA was extracted in $50 \mu l$ of elution buffer from $50 \mu l$ of each diluted virus, and tested by RT-LAMP as described above. Furthermore, the number of copies of each RNA was estimated by real-time RT-PCR using a LightCycler 480 Instrument (Roche). The reaction mixture consisted of a QuantiTect Probe RT-PCR kit (QIAGEN, Hilden, Germany), primers and a probe for detection $\mathrm{M}$ gene of influenza A viruses [Forward primer: 5'CCMAGGTCGAAACGTAYGTTCTCTCTATC-3', Reverse primer: 5'-TGACAGRATYGGTCTTGTCTTTAGCCAYTCCA-3', Probe: 5'-(FAM) ATYTCGGCTTTGAGGGGGCCTG (MGB)-3']. Five microliters of extracted RNA were added, and RT-PCR was performed at $50^{\circ} \mathrm{C}$ for $30 \mathrm{~min}, 95^{\circ} \mathrm{C}$ for $15 \mathrm{~min}$, followed by 50 cycles of $94^{\circ} \mathrm{C}$ for $15 \mathrm{sec}, 54^{\circ} \mathrm{C}$ for $75 \mathrm{sec}$ per cycle, and $40^{\circ} \mathrm{C}$ for 30 sec. The circular plasmid, M gene of A/Puerto Rico/8/1934 (H1N1) are cloned into the pHW2000 vector [8], was used for the calibration curve. The profile of fluorescence intensity during PCR was analyzed using LCS480 software (Roche).

Isolation and identification of viruses: Fecal materials of ducks were assayed as described above and inoculated into allantoic cavities of ten-day-old embryonated chicken eggs. After $48 \mathrm{hr}$ of incubation at $35^{\circ} \mathrm{C}$, allantoic fluids were tested for hemagglutinating activity. All allantoic fluids that were HA activity-positive were identified by hemagglutination inhibition (HI) and neuraminidase inhibition (NI) using specific antisera to the reference strains of influenza viruses [17]. Allantoic fluids that were HA activity-negative were inoculated again and tested for HA activity. Second allan-

Table 1. RT-LAMP primer set designed for the $\mathrm{M}$ gene of influenza A viruses

\begin{tabular}{lll}
\hline Primer name & Sequence (5'-3') & Genome position ${ }^{\text {a) }}$ \\
\hline FluAF3 & GACTTGAAGATGTCTTTGC & $80-98$ \\
FluAB3 & TGTTATTTGGATCCCCATT & $259-277$ \\
FluAFIP $(\mathrm{F} 1 \mathrm{c}+\mathrm{F} 2)^{\mathrm{b})}$ & TTAGTCAGAGGTGACAGGATTG- 149-170 (F1c)- \\
& CAGATCTTGAGGCTCTC & $110-126(\mathrm{~F} 2)$ \\
FluABIP $(\mathrm{B} 1 \mathrm{c}+\mathrm{B} 2)^{\mathrm{c})}$ & TTGTGTTCACGCTCACCGTG- & $185-204(\mathrm{~B} 1 \mathrm{c})-$ \\
& TTTGGACAAAGCGTCTACG & $226-244(\mathrm{~B} 2)$ \\
FluAFL & GTCTTGTCTTTAGCCA & $133-148$ \\
FluABL & CAGTGAGCGAGGACTG & $207-222$ \\
\hline
\end{tabular}

a) Positions of RT-LAMP primers from start codon of the $\mathrm{M}$ gene of $\mathrm{A} / \mathrm{Panama} / 2007 /$ 1999 (H3N2) (Accession number: DQ508868). b) FluAFIP primer consisted of F1c and $\mathrm{F} 2$ regions. c) FluABIP primer consisted of B1c and B2 regions. 
Table 2. Specificity of RT-LAMP for the detection of influenza viruses and avian paramyxoviruses

\begin{tabular}{|c|c|c|c|}
\hline \multirow{2}{*}{ Virus } & \multirow{2}{*}{ Lineage of the M gene ${ }^{a)}$} & \multicolumn{2}{|c|}{ RT-LAMP ${ }^{\mathrm{b})}$} \\
\hline & & Results & Threshold time \\
\hline \multicolumn{4}{|l|}{ Influenza A virus } \\
\hline Swine/Iowa/15/1930 (H1N1) & Swine & + & $11: 24$ \\
\hline Singapore/1/1957 (H2N2) & Human & + & 13:00 \\
\hline Duck/Hokkaido/5/1977 (H3N2) & Eurasian Avian & + & $12: 24$ \\
\hline Duck/Czechoslovakia/1956 (H4N6) & Eurasian Avian & + & $11: 30$ \\
\hline Swan/Hokkaido/51/1996 (H5N3) & Eurasian Avian & + & $12: 24$ \\
\hline Turkey/Massachusetts/3740/1965 (H6N2) & North American Avian & + & $14: 24$ \\
\hline Seal/Massachusetts/1/1980 (H7N7) & North American Avian & + & $12: 42$ \\
\hline Turkey/Ontario/6118/1968 (H8N4) & North American Avian & + & $13: 24$ \\
\hline Turkey/Wisconsin/1966 (H9N2) & North American Avian & + & $13: 18$ \\
\hline Chicken/Germany/N/1949 (H10N7) & Eurasian Avian & + & 14:06 \\
\hline Duck/England/1/1956 (H11N6) & Eurasian Avian & + & 13:06 \\
\hline Duck/Alberta/60/1976 (H12N5) & North American Avian & + & $13: 42$ \\
\hline Gull/Maryland/704/1977 (H13N6) & Eurasian Avian & + & $19: 36$ \\
\hline Mallard/Astrakhan/263/1982 (H14N5) & Eurasian Avian & + & $12: 42$ \\
\hline Duck/Australia/341/1983 (H15N8) & Eurasian Avian & + & $12: 24$ \\
\hline Black-headed gull/Sweden/5/1999 (H16N3) & Eurasian Avian & + & 19:12 \\
\hline \multicolumn{4}{|l|}{ Avian paramyxovirus } \\
\hline APMV-1/Miyadera & N/A & - & - \\
\hline APMV-2/chicken/Yucaipa/1956 & N/A & - & - \\
\hline APMV-3/turkey/Wisconsin/1968 & N/A & - & - \\
\hline APMV-4/duck/Mississippi/320/1975 & N/A & - & - \\
\hline APMV-5/TI & N/A & - & - \\
\hline APMV-6/duck/Hong Kong/199/1977 & N/A & - & - \\
\hline APMV-7/dove/Tennessee/4/1975 & N/A & - & - \\
\hline
\end{tabular}

a) The lineages of the M gene (Swine, Human, Eurasian Avian, and North American Avian) were identified according to the phylogenetic tree analysis. N/A; not applicable. b) Amplification of viral RNA was evaluated by LA-320C software (Eiken Chemical Co., Ltd.). +: RT-LAMP-positive, -: RT-LAMP-negative. Threshold time is the reaction time as over turbidity. Threshold Value: 0.05 was determined by LA-320C software.

toic fluids that were HA activity-negative were considered as virus isolation-negative.

Phylogenetic analysis: Viral RNA of influenza viruses listed in Table 2 were extracted from infectious allantoic fluids using a commercial kit (TRI LS reagent, Sigma) and reverse transcribed with the Uni12 primer and M-MLV Reverse Transcriptase (Invitrogen, Carlsbad, CA, U.S.A.). The full-length of the $\mathrm{M}$ gene was amplified using $\mathrm{M}$ genespecific primer set (Bm-M-1 and Bm-M-1027R) [9]. Nucleotide sequences were determined from these RT-PCR products using a 3500 Genetic Analyzer (Applied Biosystems) and analyzed by the neighbor-joining method along with those of other influenza viruses to classify the lineages of $\mathrm{M}$ genes.

\section{RESULTS}

The evaluation of sensitivity and specificity of RT-LAMP: To evaluate specificity of RT-LAMP, the assay was performed using influenza viruses and APMVs listed in Table 2. Viral RNAs of influenza A viruses of $\mathrm{H} 1$ to $\mathrm{H} 16$ subtypes were detected by RT-LAMP using the primer set of the M gene of influenza A viruses, whereas RNAs of APMVs were not detected (Table 2). From the results of phylogenetic analysis, $\mathrm{M}$ genes of these influenza viruses were classified to four lineages, Swine, Human, Eurasian Avian, and North
American Avian lineages. To evaluate the sensitivity of RT-LAMP, viral RNA in the allantoic fluids and in the fecal materials collected from ducks experimentally infected with A/duck/Hokkaido/5/1977 (H3N2), were examined for the infectivity titer of $\mathrm{EID}_{50}$, the number of viral RNA copies estimated by Real-time RT-PCR, and viral RNA detected by RT-LAMP (Table 3). For fecal materials, the detection limit of RT-LAMP was $10^{2.5}$ copies of viral RNA, which was equivalent to $10^{1.1} \mathrm{EID}_{50}$. For the infectious allantoic fluids of embryonated chicken eggs, the detection limit of RT-LAMP was $10^{2.9}$ copies of viral RNA, which was equivalent to $10^{0.9} \mathrm{EID}_{50}$.

Correlation between viral RNA detection and virus isolation from fecal materials: A total of 342 fecal materials from ducks were examined for comparative sensitivity by RTLAMP and by virus isolation using embryonated chicken eggs (Table 4). Thirty-one samples were positive for influenza viruses by RT-LAMP, and fifteen of these samples were positive by virus isolation. These isolated viruses were divided into six HA subtypes, H1, H4, H5, H6, H11, and H12. The threshold time, at which a sample was determined to be positive for influenza viruses, was within $24 \mathrm{~min}$ for the positive samples. Among 311 samples that were negative for influenza viruses on RT-LAMP, viruses with the HA activity were isolated from 12 samples. These isolates included three H4N6 influenza viruses, four APMV-1s, and 
Table 3. Sensitivity of RT-LAMP for the fecal material collected from experimentally infected ducks with A/duck/Hokkaido/5/1977 (H3N2) and the infection allantoic fluid

\begin{tabular}{|c|c|c|c|c|c|c|c|}
\hline \multirow{3}{*}{$\begin{array}{l}\text { Infectivity titer } \\
\left(\log \text { EID }_{50}\right)\end{array}$} & \multicolumn{3}{|c|}{ Fecal material $^{\text {a) }}$} & \multicolumn{4}{|c|}{ Allantoic fluid ${ }^{a)}$} \\
\hline & \multirow{2}{*}{$\begin{array}{c}\text { Genome copy number }{ }^{b)} \\
(\log )\end{array}$} & \multicolumn{2}{|c|}{ RT-LAMP') } & \multirow{2}{*}{$\begin{array}{c}\text { Infectivity titer } \\
\left(\log \text { EID }_{50}\right)\end{array}$} & \multirow{2}{*}{$\begin{array}{c}\text { Genome copy number }{ }^{\text {b) }} \\
(\log )\end{array}$} & \multicolumn{2}{|c|}{ RT-LAMP) } \\
\hline & & Results & Threshold time & & & Results & Threshold time \\
\hline 3.2 & 4.2 & + & $16: 30$ & 3.0 & 4.2 & + & $16: 30$ \\
\hline 2.9 & 3.9 & + & $17: 36$ & 2.7 & 3.9 & + & $17: 06$ \\
\hline 2.6 & 3.7 & + & $18: 54$ & 2.4 & 3.9 & + & $17: 36$ \\
\hline 2.3 & 3.4 & + & $19: 18$ & 2.1 & 3.5 & + & $19: 18$ \\
\hline 2.0 & 3.1 & + & $19: 48$ & 1.8 & 3.3 & + & $20: 48$ \\
\hline 1.7 & 2.9 & + & $23: 54$ & 1.5 & 3.1 & + & $21: 48$ \\
\hline 1.4 & 2.7 & + & $24: 42$ & 1.2 & 3.0 & + & $24: 18$ \\
\hline 1.1 & 2.5 & + & $31: 42$ & 0.9 & 2.9 & + & $24: 36$ \\
\hline 0.8 & 2.5 & - & - & 0.6 & 2.7 & - & - \\
\hline 0.5 & 2.5 & - & - & 0.3 & 2.7 & - & - \\
\hline 0.2 & 2.4 & - & - & 0.0 & 3.2 & - & - \\
\hline Negative control ${ }^{\mathrm{d})}$ & ND & - & - & Negative control ${ }^{\mathrm{d})}$ & ND & - & - \\
\hline
\end{tabular}

a) The fecal material was collected at 3 dpi from ducks experimentally infected with A/duck/Hokkaido/5/1977 (H3N2). The allantoic fluid was prepared in embryonated chicken eggs inoculated with A/duck/Hokkaido/5/1977 (H3N2). b) Average of duplicate experiments of Real-time RTPCR. ND; not detected. c) Amplification of viral RNA was evaluated by LA-320C software (Eiken Chemical Co., Ltd.). +; RT-LAMP-positive, -; RT-LAMP-negative. Threshold time is the reaction time as over turbidity. Threshold Value: 0.05 was determined by LA-320C software. d) MilliQ water was used as negative control.

five APMV-4s.

To examine the reason why influenza viruses were isolated from samples that were negative on RT-LAMP, allantoic fluids from which A/duck/Hokkaido/38/2009 (H4N6), A/duck/Hokkaido/152/2009 (H4N6), and A/duck/ Hokkaido/322/2009 (H4N6) were isolated, were directly subjected to RT-LAMP. As a result, these three isolates were positive on RT-LAMP, indicating that the discrepancy between the results of virus isolation and RT-LAMP was because virus isolation is more sensitive than RT-LAMP.

\section{DISCUSSION}

Recently, the importance of surveillance for avian influenza has been further increasing. It takes two or three days to isolate viruses using embryonated chicken eggs. Therefore, a rapid, sensitive, and specific method for detecting viruses would expect a screening procedure. In the present study, we assessed the usefulness of RT-LAMP as a screening method for detection of avian influenza virus from the fecal materials of ducks.

The primer set used in this study was designed specifically for the $\mathrm{M}$ gene, which is highly conserved and homologous within all influenza A viruses. Practically, HA subtypes and genetic background of the $\mathrm{M}$ gene of influenza viruses evaluated in this study were various, suggesting that the primer set used in this study is able to detect the M gene of multiple influenza viruses. In addition, by using loop primers (FluAFL and FluABL), viral gene is amplified efficiently and the amplification time is shortened to $35 \mathrm{~min}$ compared to previous reports $[3,12,24]$.

The detection limit of RT-LAMP using fecal material for experimentally infected ducks was $10^{2.5}$ copies of viral RNA, indicating that a very small amount of viral RNA was detectable by this assay. In the experiment using field samples, 15 influenza viruses were isolated from 31 fecal materials that were positive on RT-LAMP. Influenza viruses were isolated from samples in which viral RNA was detected by RT-LAMP, suggesting that most viruses were isolated from fecal materials containing high copy number of viral RNA. The reason why no influenza viruses were isolated from 16 positive samples by RT-LAMP is considered that complete infectious virus was not contained in fecal materials. On the other hand, three influenza viruses of H4N6 subtype were isolated from fecal materials that were negative on RT-LAMP. The nucleotide sequence of the primer set for RT-LAMP had a high homology with the $\mathrm{M}$ gene of these H4N6 viruses and viral RNA was actually amplified by RT-LAMP from allantoic fluids of embryonated chicken eggs (data not shown). Thus, it was concluded that viral RNA concentration in these fecal materials containing H4N6 viruses, which showed negative results on RT-LAMP, were less than the detection limit of RT-LAMP.

RT-LAMP appears to be a useful screening method prior to virus isolation. In addition, processing of multiple samples was available using 96-well plates for viral RNA extraction and then for RT-LAMP. Compared to previous reports $[3,12,24]$, we clearly indicated RT-LAMP as a promising tool with a high throughput capability before virus isolation using embryonated chicken eggs. We consider that using this assay as a part of the isolation procedure for influenza viruses will facilitate surveillance and timely control of avian influenza.

ACKNOWLEDGMENTS. We would like to express our sincere gratitude to the Animal Quarantine Service of the Ministry of Agriculture, Forestry and Fisheries for providing technical assistance for RT-LAMP. We also extend spe- 
Table 4. Results of RT-LAMP and virus isolation from fecal materials of ducks

\begin{tabular}{|c|c|c|c|}
\hline \multirow{2}{*}{ Sample No. } & \multicolumn{2}{|c|}{ RT-LAMP ${ }^{a)}$} & \multirow{2}{*}{$\begin{array}{l}\text { Virus } \\
\text { isolation }^{\text {b) }}\end{array}$} \\
\hline & Results & Threshold time & \\
\hline 1 & + & $15: 06$ & - \\
\hline 2 & + & $15: 36$ & - \\
\hline 3 & + & $16: 00$ & AIV (H1N5) \\
\hline 4 & + & $16: 00$ & AIV (H6N1) \\
\hline 5 & + & $16: 00$ & AIV (H11N9) \\
\hline 6 & + & $16: 06$ & AIV (H11N9) \\
\hline 7 & + & $16: 12$ & AIV (H6N1) \\
\hline 8 & + & $16: 12$ & AIV (H6N1) \\
\hline 9 & + & $16: 24$ & AIV (H11N9) \\
\hline 10 & + & $17: 18$ & AIV (H12N5) \\
\hline 11 & + & $17: 18$ & - \\
\hline 12 & + & $18: 30$ & AIV (H1N3) \\
\hline 13 & + & $18: 42$ & AIV (H6N8) \\
\hline 14 & + & $18: 48$ & AIV (H6N8) \\
\hline 15 & + & $20: 12$ & - \\
\hline 16 & + & $20: 18$ & AIV (H5N1) \\
\hline 17 & + & $20: 24$ & AIV (H4N6) \\
\hline 18 & + & $20: 24$ & AIV (H6N1) \\
\hline 19 & + & $22: 18$ & - \\
\hline 20 & + & $23: 48$ & AIV (H4N6) \\
\hline 21 & + & $24: 30$ & - \\
\hline 22 & + & $25: 54$ & - \\
\hline 23 & + & $26: 54$ & - \\
\hline 24 & + & $27: 30$ & - \\
\hline 25 & + & $27: 30$ & - \\
\hline 26 & + & $27: 42$ & - \\
\hline 27 & + & $27: 54$ & - \\
\hline 28 & + & $30: 00$ & - \\
\hline 29 & + & $30: 54$ & - \\
\hline 30 & + & $32: 00$ & - \\
\hline 31 & + & $32: 30$ & - \\
\hline 32 & - & - & AIV (H4N6) \\
\hline 33 & - & - & AIV (H4N6) \\
\hline 34 & - & - & AIV (H4N6) \\
\hline 35 & - & - & APMV-1 \\
\hline 36 & - & - & APMV-1 \\
\hline 37 & - & - & APMV-1 \\
\hline 38 & - & - & APMV-1 \\
\hline 39 & - & - & APMV-4 \\
\hline 40 & - & - & APMV-4 \\
\hline 41 & - & - & APMV-4 \\
\hline 42 & - & - & APMV-4 \\
\hline 43 & - & - & APMV-4 \\
\hline
\end{tabular}

Other 299 samples

a) Amplification of viral RNA was evaluated by LA-320C software (Eiken Chemical Co., Ltd.). +; RT-LAMP-positive, -; RT-LAMPnegative. Threshold time is the reaction time as over turbidity. Threshold Value: 0.05 was determined by LA-320C software. b) AIV; Avian influenza virus, APMV-1; Avian paramyxovirus type 1, APMV-4; Avian paramyxovirus type 4. HA and NA subtypes of isolated AIVs were indicated in parentheses. -; Virus isolationnegative.

cial thanks to the Nippon Institute for Biological Science for providing the APMV type-5 TI strain. The present work was supported by the Program of Founding Research Centers for Emerging and Reemerging Infectious Diseases from the Ministry of Education, Culture, Sports, Science and Technology, Japan.

\section{REFERENCES}

1. Banks, J., Speidel, E. S., Moore, E., Plowright, L., Piccirillo, A., Capua, I., Cordioli, P., Fioretti, A. and Alexander, D. J. 2001. Changes in the haemagglutinin and the neuraminidase genes prior to the emergence of highly pathogenic $\mathrm{H} 7 \mathrm{~N} 1$ avian influenza viruses in Italy. Arch. Virol. 146: 963-973.

2. Chen, H., Smith, G. J., Zhang, S. Y., Qin, K., Wang, J., Li, K. S., Webster, R. G., Peiris, J. S. and Guan, Y. 2005. Avian flu: H5N1 virus outbreak in migratory waterfowl. Nature $\mathbf{4 3 6}$ : 191-192.

3. Chen, H. T., Zhang, J., Sun, D. H., Ma, L. N., Liu, X. T., Cai, X. P. and Liu, Y. S. 2008. Development of reverse transcription loop-mediated isothermal amplification for rapid detection of H9 avian influenza virus. J. Virol. Methods 151: 200-203.

4. Compton, J. 1991. Nucleic acid sequence-based amplification. Nature 350: 91-92.

5. Fouchier, R. A., Munster, V., Wallensten, A., Bestebroer, T. M., Herfst, S., Smith, D., Rimmelzwaan, G. F., Olsen, B. and Osterhaus, A. D. 2005. Characterization of a novel influenza A virus hemagglutinin subtype (H16) obtained from blackheaded gulls. J. Virol. 79: 2814-2822.

6. Guatelli, J. C., Whitfield, K. M., Kwoh, D. Y., Barringer, K. J., Richman, D. D. and Gingeras, T. R. 1990. Isothermal, in vitro amplification of nucleic acids by a multienzyme reaction modeled after retroviral replication. Proc. Natl. Acad. Sci. U.S.A. 87: 1874-1878.

7. Higuchi, R., Dollinger, G., Walsh, P. S. and Griffith, R. 1992. Simultaneous amplification and detection of specific DNA sequences. Biotechnology (N.Y.) 10: 413-417.

8. Hoffmann, E., Neumann, G., Kawaoka, Y., Hobom, G. and Webster, R. G. 2000. A DNA transfection system for generation of influenza A virus from eight plasmids. Proc. Natl. Acad. Sci. U.S.A. 97: 6108-6113.

9. Hoffmann, E., Stech, J., Guan, Y., Webster, R. G. and Perez, D. R. 2001. Universal primer set for the full-length amplification of all influenza A viruses. Arch. Virol. 146: 2275-2289.

10. Horimoto, T., Rivera, E., Pearson, J., Senne, D., Krauss, S., Kawaoka, Y. and Webster, R. G. 1995. Origin and molecular changes associated with emergence of a highly pathogenic H5N2 influenza virus in Mexico. Virology 213: 223-230.

11. Imai, M., Ninomiya, A., Minekawa, H., Notomi, T., Ishizaki, T., Tashiro, M. and Odagiri, T. 2006. Development of H5-RTLAMP (loop-mediated isothermal amplification) system for rapid diagnosis of $\mathrm{H} 5$ avian influenza virus infection. Vaccine 24: 6679-6682.

12. Ito, M., Watanabe, M., Nakagawa, N., Ihara, T. and Okuno, Y. 2006. Rapid detection and typing of influenza A and B by loop-mediated isothermal amplification: comparison with immunochromatography and virus isolation. J. Virol. Methods 135: 272-275.

13. Ito, T., Goto, H., Yamamoto, E., Tanaka, H., Takeuchi, M., Kuwayama, M., Kawaoka, Y. and Otsuki, K. 2001. Generation of a highly pathogenic avian influenza A virus from an avirulent field isolate by passaging in chickens. J. Virol. 75: 44394443.

14. Ito, T., Okazaki, K., Kawaoka, Y., Takada, A., Webster, R. G. and Kida, H. 1995. Perpetuation of influenza A viruses in Alaskan waterfowl reservoirs. Arch. Virol. 140: 1163-1172. 
15. Kawaoka, Y. and Webster, R. G. 1985. Evolution of the A/ Chicken/Pennsylvania/83 (H5N2) influenza virus. Virology 146: $130-137$.

16. Kida, H., Kawaoka, Y., Naeve, C. W. and Webster, R. G. 1987. Antigenic and genetic conservation of $\mathrm{H} 3$ influenza virus in wild ducks. Virology 159: 109-119.

17. Kida, H. and Yanagawa, R. 1979. Isolation and characterization of influenza a viruses from wild free-flying ducks in Hokkaido, Japan. Zentralbl. Bakteriol. Orig. A 244: 135-143.

18. Kida, H., Yanagawa, R. and Matsuoka, Y. 1980. Duck influenza lacking evidence of disease signs and immune response. Infect. Immun. 30: 547-553.

19. Manzoor, R., Sakoda, Y., Mweene, A., Tsuda, Y., Kishida, N., Bai, G. R., Kameyama, K., Isoda, N., Soda, K., Naito, M. and Kida, H. 2008. Phylogenic analysis of the M genes of influenza viruses isolated from free-flying water birds from their Northern Territory to Hokkaido, Japan. Virus Genes 37: 144-152.

20. Notomi, T., Okayama, H., Masubuchi, H., Yonekawa, T., Watanabe, K., Amino, N. and Hase, T. 2000. Loop-mediated isothermal amplification of DNA. Nucleic Acids Res. 28: E63.

21. OIE. 2010. Facts \& Figures: Avian Influenza H5N1 notified in 2003-2010 [cited 2010 September 1]. Available from http:// www.oie.int/eng/info_ev/en_AI_factoids_2.htm

22. Okazaki, K., Takada, A., Ito, T., Imai, M., Takakuwa, H., Hatta, M., Ozaki, H., Tanizaki, T., Nagano, T., Ninomiya, A., Demenev, V. A., Tyaptirganov, M. M., Karatayeva, T. D., Yamnikova, S. S., Lvov, D. K. and Kida, H. 2000. Precursor genes of future pandemic influenza viruses are perpetuated in ducks nesting in Siberia. Arch. Virol. 145: 885-893.

23. Sakoda, Y., Sugar, S., Batchluun, D., Erdene-Ochir, T. O., Okamatsu, M., Isoda, N., Soda, K., Takakuwa, H., Tsuda, Y.,
Yamamoto, N., Kishida, N., Matsuno, K., Nakayama, E., Kajihara, M., Yokoyama, A., Takada, A., Sodnomdarjaa, R. and Kida, H. 2010. Characterization of H5N1 highly pathogenic avian influenza virus strains isolated from migratory waterfowl in Mongolia on the way back from the southern Asia to their northern territory. Virology 406: 88-94.

24. Shivakoti, S., Ito, H., Murase, T., Ono, E., Takakuwa, H., Yamashiro, T., Otsuki, K. and Ito, T. 2010. Development of reverse transcription-loop-mediated isothermal amplification (RT-LAMP) assay for detection of avian influenza viruses in field specimens. J. Vet. Med. Sci. 72: 519-523.

25. Silvano, F. D., Yoshikawa, M., Shimada, A., Otsuki, K. and Umemura, T. 1997. Enhanced neuropathogenicity of avian influenza A virus by passages through air sac and brain of chicks. J. Vet. Med. Sci. 59: 143-148.

26. Uchida, Y., Mase, M., Yoneda, K., Kimura, A., Obara, T., Kumagai, S., Saito, T., Yamamoto, Y., Nakamura, K., Tsukamoto, K. and Yamaguchi, S. 2008. Highly pathogenic avian influenza virus (H5N1) isolated from whooper swans, Japan. Emerg. Infect. Dis. 14: 1427-1429.

27. Walker, G. T., Fraiser, M. S., Schram, J. L., Little, M. C., Nadeau, J. G. and Malinowski, D. P. 1992. Strand displacement amplification - an isothermal, in vitro DNA amplification technique. Nucleic Acids Res. 20: 1691-1696.

28. Walker, G. T., Little, M. C., Nadeau, J. G. and Shank, D. D. 1992. Isothermal in vitro amplification of DNA by a restriction enzyme/DNA polymerase system. Proc. Natl. Acad. Sci. U.S.A. 89: 392-396.

29. Webster, R. G., Bean, W. J., Gorman, O. T., Chambers, T. M. and Kawaoka, Y. 1992. Evolution and ecology of influenza A viruses. Microbiol. Rev. 56: 152-179. 